\title{
Multi-scale Modelling of Carbon Nanotube Reinforced Crosslinked Interfaces
}

\author{
Elif Ozden-Yenigun, ${ }^{* a}$ Canan Atilgan ${ }^{b}$ and James A. Elliott ${ }^{c}$ \\ ${ }_{5}^{a}$ Textile Engineering, Faculty of Textile Technologies and Design, Istanbul Technical University, \\ Istanbul, 34437 Turkey; ozdenyenigun@itu.edu.tr \\ ${ }^{b}$ Department of Materials Science \& Engineering, Faculty of Engineering and Natural Sciences, Sabanci \\ University, Istanbul 34956, Turkey; canan@sabanciuniv.edu \\ ${ }^{c}$ Department of Materials Science, University of Cambridge, 27 Charles Babbage Road, CB3 OFS, \\ 10 United Kingdom; jae1001@cam.ac.uk
}

\begin{abstract}
In this paper, we study the crosslinking route and interface interactions for achieving superior properties in carbon nanotube (CNT)-reinforced epoxy based nanocomposites by using multi${ }_{15}$ scale modelling. For that purpose, polymeric epoxy matrices consisting of EPON 862 epoxy and TETA hardener molecules were coarse-grained and simulated using the dissipative particle dynamics (DPD) method. Furthermore, CNTs were coarse-grained as rigid rods and embedded into the uncrosslinked mesoscopic polymer system. Reverse-mapping of the atomistic details onto coarse-grained models was carried out to allow further simulations at the atomistic scale ${ }_{20}$ using molecular dynamics (MD) by keeping the periodicity of the CNTs structure. The mechanism of crosslinking is simulated, and both neat and CNTs-reinforced thermoset nanocomposites with different degrees of crosslinking were reconstructed. Normal stresses in tensile and compressive loading direction up to $0.2 \%$ strain were calculated and yield strength ( $0.2 \%$ offset) and compressive/elastic modulus in both normal directions were reported, which ${ }_{25}$ match the experimental values as well. Overall, this paper explores a fast and straightforward procedure to bridge periodic mesoscopic structures such as CNTs and their nanocomposites to experimentally tested material properties.
\end{abstract}

Keywords: epoxy, carbon nanotubes, molecular dynamics, reverse mapping, dissipative particle dynamics

\section{Introduction}

${ }_{30}$ The interfacial adhesion between filler and matrix in polymer nanocomposites is crucial to determining their properties, and difficult to investigate experimentally. Computational modelling offers a tool to investigate how strength and stiffness of the composite depend on the degree of cross-linking in the matrix and reinforcement provided by nanofillers. Due to their excellent mechanical and thermal stability neat thermoset epoxy resins have been investigated thoroughly in the literature, and as a matrix, they have been simulated extensively by molecular dynamics (MD) ${ }^{1-18}$. For a proper understanding of their ${ }_{35}$ equilibrated structures, there are several attempts which perform coarse-graining to simulate these crosslinked networks on larger time and length scales ${ }^{2,19,20}$. Researchers have also been interested in epoxy based nanocomposites and investigated the effect of nanofillers on crosslinked networks ${ }^{7,21-31}$. Reinforced epoxy resins exhibit many superior properties, including mechanical behaviour which can be modified by an appropriate matrix-filler interaction. However, poor interactions between the matrix and nanofillers are one of the key limiting factors preventing. Previous computational studies on the interface 40 between filler (or substrate) and epoxy resins have focused on providing insight to failure and deformation mechanisms $\mathrm{s}^{7,19}$, 24, 32-35. Not only the effect of reinforcement but also that of crosslinking density in epoxy resins which has strong influence on mechanical properties have been investigated ${ }^{9}$. However, to implement solely MD simulations to predict mechanical properties may be challenging due to the requirement of larger representative simulation boxes and extremely long simulation times to obtain well-relaxed atomistic structures, particularly in the presence of nanofillers such as carbon nanotubes (CNTs).

${ }_{45}$ For instance, in our previous study ${ }^{36}$, representative crosslinked units were used to create highly crosslinked epoxy resin. However, insufficient relaxation of the structures occurred for proper comparison with experimentally reported properties. Nevertheless, multi-scale methods are rarely employed in epoxy systems in literature. Recently, Kacar et al. ${ }^{37}$ predicted material properties of a reverse-mapped neat cross-linked polymer by using multi-scale simulations and compared the elastic properties and glass transition temperature with experimental values. Even though, an efficient and reliable reverse-mapping ${ }_{50}$ procedurs was reported for crosslinked systems, the effect of nanofillers on the crosslinking mechanism was still not considered. 
Herein, we propose a novel multi-scale numerical simulation method for nanocomposites, which can be, applied both to thermoset and thermoplastic matrices. Using this method, crosslinking reaction in the presence of nanofillers is also considered.

First, as described in section DPD parametrization, a coarse-grained parameterization was developed to study the ${ }_{5}$ nanocomposites on longer length scales. Next, a reverse-mapping process was carried out to project atomistic structure back onto the coarse-grained system. Consecutive numerical simulations at different length-scales allow prediction of long-term behaviour of polymer matrices where direct MD simulations are not computationally efficient. Besides, to the best of our knowledge, this is the first time periodic structures such as carbon nanotubes were coarse-grained as rigid rods and then reverse-mapped by keeping the periodicity of the structure. Then, as described in the section Crosslinking Algorithm, the 10 crosslinking mechanism was simulated and thermoset matrices containing EPON 862 and TETA hardener molecules with different crosslinking degrees were reconstructed from the reverse-mapped structures. The structural evolution was monitored during crosslinking, both in neat and CNT reinforced epoxy systems. The mechanical properties of nanocomposites were studied at the molecular level to explore optimum process and materials parameters. Finally, structural-property relationships by varying curing degree were investigated in the section Mechanical Properties. It is found that both tensile/compressive is strength and modulus gradually increase with an increasing extent of reaction. Surprisingly, even though the incorporation of CNTs into resin systems changed the crosslinking scheme and reduced the degree of crosslinking in epoxy matrix, embedding CNTs into epoxy systems increased both modulus and strength of the polymer network.

\section{Materials and Methods}

\section{${ }_{20} 2.1$ Computational Methodology}

\subsubsection{DPD Parameterization}

DPD simulations were performed on a collection of particles, each made up of a suitably selected set of atoms (Figure 1) EPON 862 has three different beads labelled bead A, $B_{1}$ and $B_{2}$. Bead A has reactive epoxide rings. Meanwhile, SWNT $(6,6)$ and TETA molecules were composed of repeating single bead structures, and named as $\mathrm{C}_{6}$ and $\mathrm{C}$, respectively. The force ${ }_{25}$ acting on a given DPD bead at each time step is the sum of soft repulsive forces acting along the line connecting the bead to its neighbours, a dissipative term and a random force term. In addition, the beads that are located consecutively along the contour of a chain are connected by a harmonic spring. In a DPD simulation, the chains need to be partitioned into beads made up of chemically distinct units that also have similar sizes. The units we use in the current study are displayed in Figure 1a and Figure 1b, where $\mathbf{A}, \mathbf{B}_{\mathbf{1}}, \mathbf{B}_{2}$ represent the epoxy and $\mathbf{C}$ represents hardener segments. Meanwhile, considering the ${ }_{30}$ regular pattern of single walled carbon nanotubes (SWNT), SWNT $(6,6)$ nanostructures were coarse-grained based on the motion groups shown in Figure 1c. Herein, we used motion groups and their default point of rotation, its center of mass, as pattern of coarse-grained structure. Besides, this analogy speeds up the calculation since a motion group which is a collection of particles that can be translated or rotated such that the coordinates of each particle in the group remain fixed relative to the coordinates of all the other particles in the group.

${ }_{35}$ Following established protocol ${ }^{38}$, Hildebrand solubility parameters ${ }^{39}$ were calculated from successive 1 ps equilibration step and $100 \mathrm{ps} \mathrm{MD}$ on simulation boxes that contain 10 beads of the same type with a density of $1.0 \mathrm{~g} \mathrm{~cm}^{-3}$ at $298 \mathrm{~K}$, using the Amorphous Cell and Forcite modules of Materials Studio $7.0^{\circledR 40}$. For all non-bonded interactions, a cut-off radius, $r_{c}$, of 8.5 $\AA$ and periodic boundary conditions were applied in the canonical ensemble (NVT). The molar volume of the beads, $V_{m}$, was calculated using ACDLabs/Chemsketch 5. $0^{41}$. Solubility parameters were determined according to:

40

$$
\delta=\left(\Delta E v / V_{m}\right)^{1 / 2}=(C E D)^{1 / 2},
$$

where $\Delta E_{v}$ and CED correspond to molar energy of vaporization and cohesive energy density, respectively. The FloryHuggins interaction parameters, $\chi_{i, j}$, for each pair of beads were then calculated from:

$$
\chi_{i j}=\frac{V_{m}}{\mathrm{k}_{\mathrm{B}} \mathrm{T}}\left(\delta_{i}-\delta_{j}\right)^{2},
$$

where $V_{m}$ is the average molar volume of beads $i$ and $j$. The calculated values of $\delta$ and $\chi$ are displayed in Table 1 .

${ }_{45}$ The Flory-Huggins parameter, $\chi$ is related to the thermodynamics of mixing. The DPD interactions parameters, $a_{i j}$, obtained from $\chi$, is a measure of the repulsion between the beads. The latter are calculated using the linear relationship put forth by Groot and Warren ${ }^{38}$ as $a_{i i}=25 k_{b} T$ and $a_{i j} \approx a_{i i}+3.27 \chi_{i j}$ for a box density of 3 DPD units (Table 1). In addition, carbon nanotubes were treated as rigid rods with a high spring constant and repulsive interaction parameters were assigned. We note that this treatment assumes equal repulsive interactions between like beads which is strictly true only when the molar volumes ${ }_{50}$ of the two components are equal ${ }^{42}$. In this work, we are interested in finding the long-time evolution of the coordinates of each segment by tracking the centroids of beads. To obtain well-equilibrated crosslinked systems in molecular detail, reversemapping was executed at a later stage, described in section Reverse-Mapping Methodology. 
Table 1 Properties of beads defined in Fig.1. Solubility parameters, $\delta$, molar volumes $\mathrm{V}_{\mathrm{m}}$, Flory-Huggins interaction parameters, $\chi \mathrm{ij}$, (upper diagonal, shown in bold) and DPD interaction parameters, aij (lower diagonal).

\begin{tabular}{cccccc}
\hline & $\mathbf{A}$ & \multicolumn{1}{c}{$\mathbf{B}_{\mathbf{1}}$} & $\mathbf{B}_{2}$ & $\mathbf{C}$ & $\mathbf{C 6}$ \\
$\delta\left(\mathrm{cal} \mathrm{cm}^{-3}\right)^{1 / 2}$ & 12.4 & 9.58 & 9.99 & 12.6 & 7.03 \\
& 6 & & & 6 & \\
$V_{m}\left(\mathrm{~cm}^{3} \mathrm{~mol}^{-1}\right)$ & 62.8 & 105. & 89.4 & 90.5 & 89.4 \\
& \multicolumn{5}{c}{7} \\
\hline & $\mathbf{0 . 0 0}$ & $\mathbf{1 . 1 8}$ & $\mathbf{0 . 7 8}$ & $\mathbf{0 . 0 1}$ & $\mathbf{4 . 5 9}$ \\
$\mathbf{A}$ & 25.0 & & & & \\
& 0 & & & & \\
& & $\mathbf{0 . 0 0}$ & $\mathbf{0 . 0 3}$ & $\mathbf{1 . 5 7}$ & $\mathbf{4 . 5 9}$ \\
$\mathbf{B}_{1}$ & 25.8 & 25.0 & & & \\
& 6 & 0 & & & \\
& & & $\mathbf{0 . 0 0}$ & $\mathbf{1 . 0 8}$ & $\mathbf{4 . 5 9}$ \\
$\mathbf{B}_{2}$ & 27.5 & 25.1 & 25.0 & & \\
& 6 & 0 & 0 & & \\
& & & & $\mathbf{0 . 0 0}$ & $\mathbf{4 . 5 9}$ \\
$\mathbf{C}$ & 25.0 & 30.1 & 28.5 & 25.0 & \\
& 1 & 4 & 4 & 0 & \\
& & & & & $\mathbf{0 . 0 0}$ \\
$\mathbf{C 6}$ & 40.0 & 40.0 & 40.0 & 40.0 & 25.0 \\
& 0 & 0 & 0 & 0 & 0 \\
\hline
\end{tabular}

\subsubsection{DPD Simulations}

For DPD simulations, epoxy, hardener molecules and CNTs were coarse-grained via a pattern document by maintaining the initial configuration of each molecule, shown in Figure 1. This pattern document did the matching of the names of beads and motion groups assigned in molecular structure to successively complete coarse-graining. Different bead names and motion ${ }_{10}$ groups were also assigned to each structure at this step. Later on, this structural information is used in the reverse-mapping algorithm. Cubic boxes having $30 \times 30 \times 30 u^{3}$ volume were constructed with a density of 3 DPD units where $u$ is the cut-off radius. A harmonic spring constant of 200 (in DPD units) was chosen for polymer and rigid nanotube beads located consecutively along the structure, respectively. The temperature and bead masses were taken as unity and time step was set to $\approx 0.254$ ps $(\approx 0.05$ in reduced units $)$; the conversion is calculated through the Mesocite module. Following earlier studies ${ }^{1}$, ${ }_{15}{ }^{43}$ which propose optimal weight ratio (100:15.4) for epoxy and curing agent, the epoxy: hardener ratio was adjusted to 3:1. In this manner, the composition of CNT-reinforced uncrosslinked epoxy system was also set. The systems were equilibrated for 20,000 DPD time steps, followed by 100,000 DPD time steps.

\subsubsection{Reverse-Mapping Methodology}

${ }_{20}$ Several methods ${ }^{44-52}$ have been proposed for the reverse-mapping of coarse-grained model into a detailed atomistic model. In this study, a general strategy in line with Marrink et al. ${ }^{50}$ was adopted to reconstruct low energy atomistic structures. The reverse-mapping algorithm was executed via scripting implemented in the Materials Studio software $7.0^{40}$. This algorithm significantly reduces data processing times and enables the reconstruction of larger polymer systems. Two consecutive steps have been carried out to generate the atomistic ensemble that underlies its corresponding well-relaxed coarse-grained system.

${ }_{25}$ First, atomistic molecules were positioned close to their reference coarse-grained particles by matching motion groups and centroids. The second step was to relax the constructed reverse-mapped systems which follows the successive route of (i) optimization, (ii) MD with a cooling cycle (from $1500 \mathrm{~K}$ to $300 \mathrm{~K}$ ) with distance between neighbouring centroids fixed by restraints $\left(100 \mathrm{kcal} \mathrm{mol}^{-1} \AA^{-2}\right)$ and (iii) constant temperature MD with decreasing restraint energy (See Figure S1 for the temperature profile of pure EPON862 system during this procedure). As the assigned restraints between centroids were ${ }_{30}$ gradually removed, relaxed reverse-mapped structures were achieved. (Figure S2). CED analysis on these systems, calculated as potential energy $E_{p o t}$ per unit volume of selected groups of molecules, demonstrated that systems for which MD simulations were carried out after reverse-mapping (RM-MD) had lower energy profiles than those that were obtained via direct-MD simulations (Figure S3) 


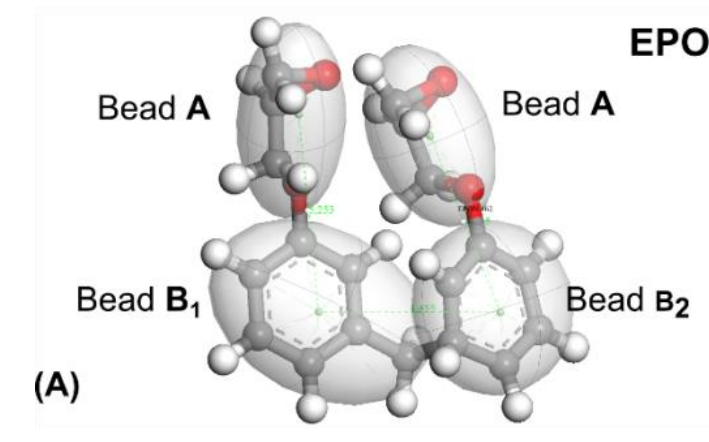

EPON 862

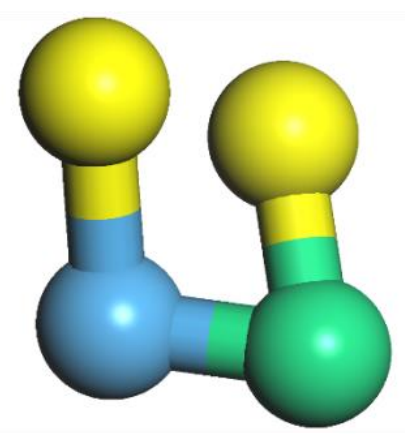

TETA

(B)
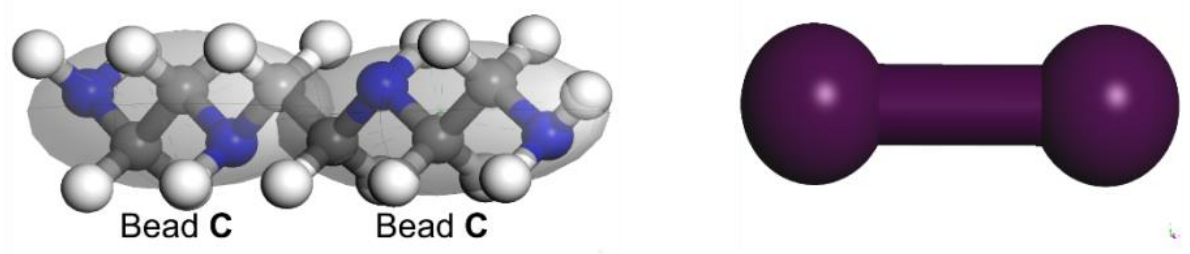

(C)

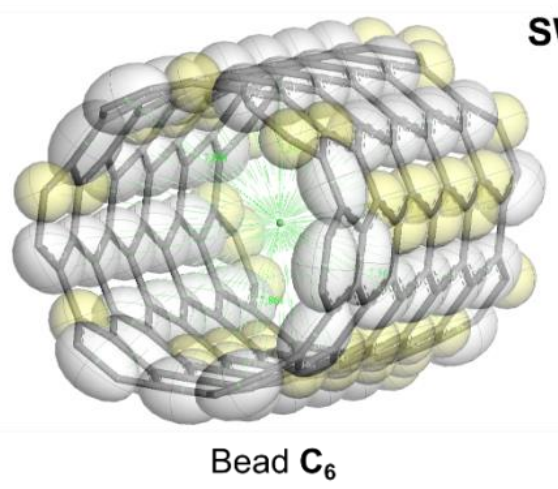

$\operatorname{SWNT}(6,6)$

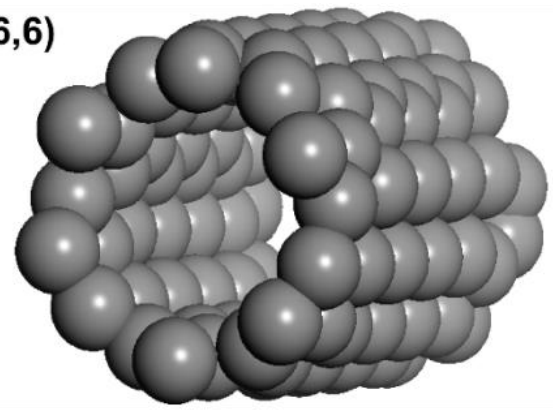

(D)

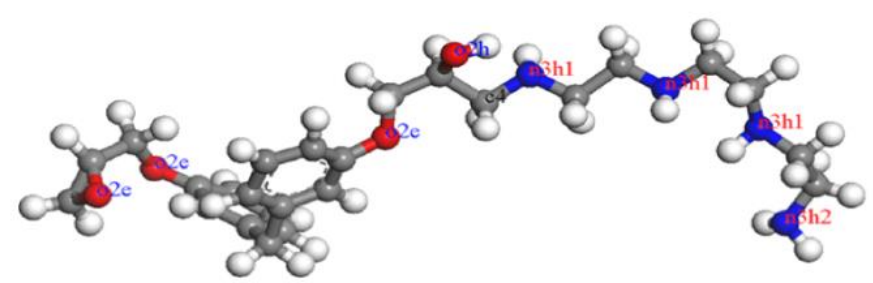

Fig. 1 Atomistic ball-stick model and coarse-grained model representations of (A) epoxy (EPON 862) (B) hardener (TETA) molecules and (C) SWNT(6,6) structures. Partitioning of the beads $\left(\mathbf{A}, \mathbf{B}_{1}, \mathbf{B}_{2}, \mathbf{C}\right.$ and $\left.\mathbf{C}_{\mathbf{6}}\right)$ for coarse-grained simulations are also displayed. (D)

Representative EPON 862-TETA crosslinked chains with reactive sites, labelled using the COMPASS force field notation

\subsubsection{Crosslinking Algorithm}

There are two approaches to obtain crosslinked networks: (i) to use representative crosslinked units with a certain degree of crosslinking, then performing classical MD runs for equilibration and data collection ${ }^{36,53}$ (ii) to create a simulation box including primary molecules of resin and curing agent without any crosslink, then performing a cyclic set of minimization, 10 equilibration and dynamics runs to reach a crosslinked structure by forming a chemical bond with a probability depending on the reactivity of groups whenever two atoms participating in a crosslink are close in space ${ }^{2,9,12}$.Our previous studies ${ }^{36,53}$ indicated that the first approach is ineffective in the construction of 3D complex structures such as epoxy networks due to the equilibration problems and the associated computational cost. Moreover, the effect of nanofillers (nanofibers or 
nanotubes) on crosslinking is disregarded through this method. Even though the second method is more complicated and time consuming in large systems, it is preferred due to its accuracy and flexibility in tuning reaction parameters.

We propose an effective reverse-mapping algorithm which can be used to construct both large thermoset and thermoplastic matrices. Herein, we explored a crosslinking algorithm that is capable of constructing highly crosslinked epoxy networks and ${ }_{5}$ predicting the physical properties in close agreement with experimental measurements. EPON 862 is a bi-functional reactant with two epoxide groups at two ends, while TETA has four reactive sites including both primary and secondary amine groups, and hence is a multifunctional reactant (Figure 1). Therefore, EPON 862 and TETA are able to produce complex 3D crosslinked epoxy polymers. The $\mathrm{C}-\mathrm{O}$ bond in each epoxide group needs to be broken in order to form a reactive $-\mathrm{CH}_{2}$ site, capable of being crosslinked to a TETA molecule.

${ }_{10}$ There are two primary amines $\left(-\mathrm{NH}_{2}\right)$ and two secondary amines $(-\mathrm{NH}-)$ in TETA molecules. The tendency of primary and secondary amines to participate in the crosslinking reaction can be varied depending on the reaction conditions. At relatively low temperatures, the activation energy of primary reactions is lower than that of secondary amines; the difference in activation energies can be negligible at sufficiently high temperatures ${ }^{54}$. In this part of the study, a fixed temperature of 500 $\mathrm{K}$ was used for MD. Throughout the crosslinking reaction at $500 \mathrm{~K}$, an equal reactivity ratio was assigned for each $\mathrm{N}-\mathrm{H}$ group 15 and two different crosslinking routes were studied. First, a curing agent with two reactive sites (primary amines only, $n 3 h 2$ in Condensed-phase Optimized Molecular Potentials for Atomistic Simulation Studies (COMPASS) notation) underwent reaction and the crosslinking density of this system was evaluated. To create a highly crosslinked network, both primary (n3h2) and secondary $(n 3 h 1)$ amines were defined as reactive sites. Figure 1d displays representative EPON862-TETA crosslinked unit their reactive sites, labelled in COMPASS notation. A three-step crosslinking procedure including pre${ }_{20}$ equilibration, creation of covalent bonds and post-equilibration was completed. These two different crosslinking paths were followed also in the presence of CNT to form CNT reinforced epoxy networks. Close contacts (within $4.5 \AA$ of each other) were identified between the reactive atoms and new bonds were created with probability $p$ if the distance between these two atoms is equal or less than reaction distance, where $p$ is the reactivity ratio. Once new connections were built, hydrogen atoms were reset. Post equilibration was performed to relax the atomistic structure.

25

\subsubsection{Simulations of Reverse-mapped Structures}

MD simulations provide an atomistic level picture of structure and dynamics. As in the case of crosslinked networks, MD simulations allow the prediction of properties of materials that rely on atomistic detail as well as interpreting their mechanical behaviour. In this study, we use MD simulations to perform structural analysis and to calculate mechanical properties of ${ }_{30}$ reinforced and unreinforced epoxy systems. The molecular simulation software package Materials Studio ${ }^{\circledR} 7.0^{40}$ was used to construct the initial molecular structures, simulations and post-processing the collected trajectories. The COMPASS (Condensed-phase Optimized Molecular Potentials for Atomistic Simulation Studies) force field ${ }^{55}$ used in this study has been shown to be very effective in defining properties of synthetic polymers and epoxy. MD simulations ( $5 \mathrm{~ns})$ of reverse-mapped systems were carried out in the isothermal-isobaric (NPT) statistical ensemble, at $P=1 \mathrm{~atm}$ and $T=300 \mathrm{~K}$. To maintain ${ }_{35}$ temperature and pressure fixed at their prescribed values, the Andersen/Berendsen thermostat/barostat were used ${ }^{56,57}$. Detailed structural and mechanical analyses were performed on the equilibrated reinforced and unreinforced systems. We note that control runs of $20 \mathrm{~ns}$ were performed on highly crosslinked neat systems where the systems were directly constructed instead of relaxing them through DPD simulations followed by the reverse mapping procedure. 

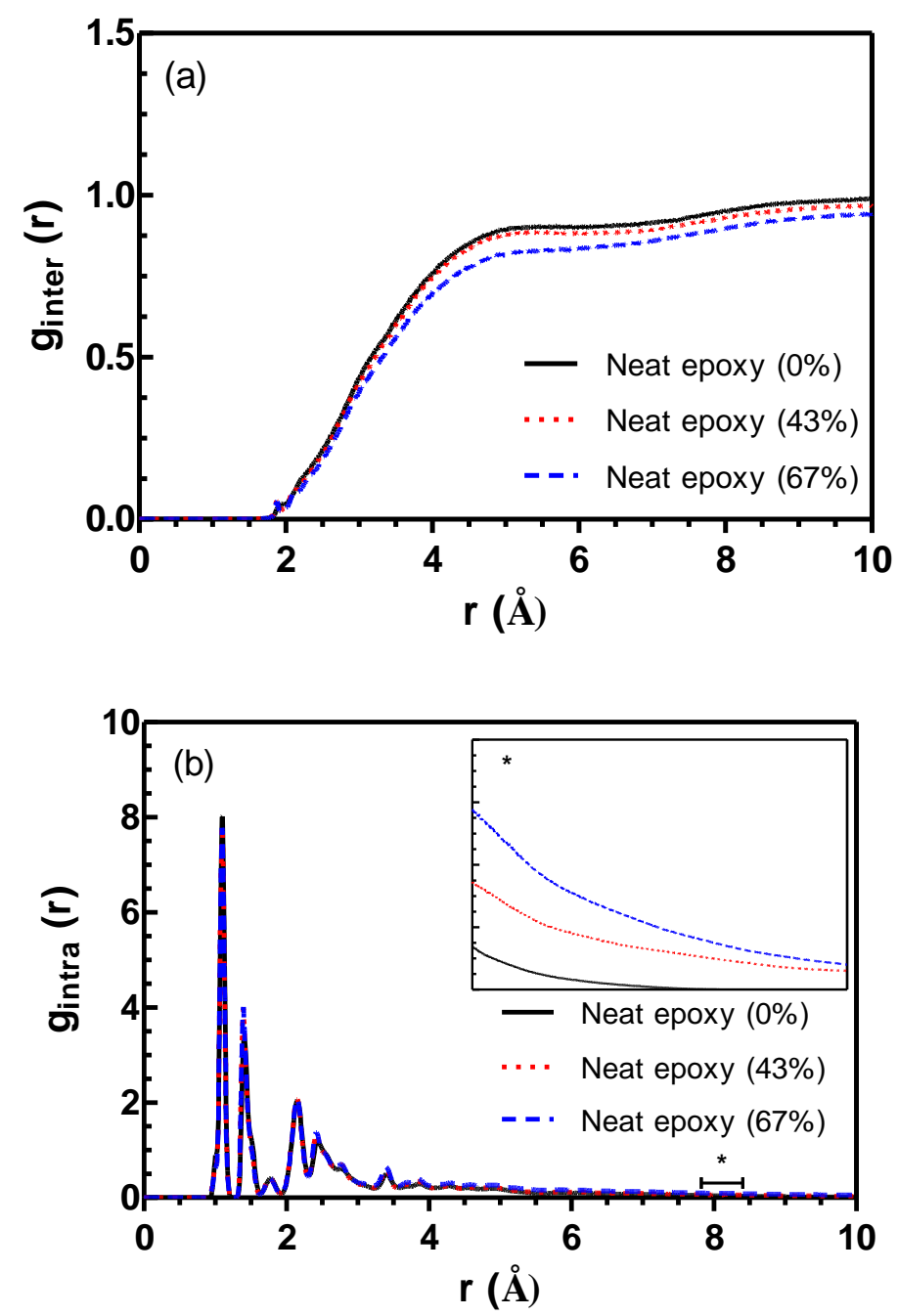

Fig. 2 (a) Inter- and (b) intra-molecular RDFs of all atoms for: unreinforced epoxy systems at $0 \%$, at $43 \%$ and at $67 \%$ crosslinking densities

\section{s 2.1.5 Calculation of Mechanical Properties}

Several approaches such as static ${ }^{58}$, dynamical ${ }^{59}$ and fluctuation methods ${ }^{60,61}$ can be used for the calculation of mechanical properties. However, in order to obtain reasonably precise estimates, the fluctuation methods ${ }^{60,61}$ tend to be relatively slow. Besides, incorporation of CNTs and their effect on the crosslinking path may lead to anisotropy. Thus, the isotropic material assumption might not be representative and accurate enough to obtain elastic constants of reinforced systems. Therefore, in 10 this study, mechanical behaviour of reinforced and unreinforced systems was evaluated via the application of incremental strains using a scripting approach: Stress states associated with loading were obtained from the deformation of the simulation cell. For each strain level, the unit cell was compressed along one dimension (e.g. the $y$-direction) and the other two dimensions (e.g. $x$ and $z$-directions) were extended to maintain the constant density; in each compression step, the periodic cell was post-equilibrated ( $200 \mathrm{ps})$ using the new dimensions. The stresses were averaged in the normal and shear directions 15 over the second half of the equilibration run. Stress tensors (with elements $\tilde{o}_{i j}$ ) of reinforced and unreinforced systems up to $0.2 \%$ strain were obtained and interpreted to calculate elastic modulus and compressive modulus from normal directions.

\section{Results and Discussion}

\subsection{Local Structure Evolution of Neat Crosslinked Networks.}

${ }_{20}$ To study the local structural evolution of uncrosslinked and crosslinked polymers and investigate the effect of crosslinking and reinforcement on the structure and properties of epoxies, radial distribution functions (RDFs) of different pairs of atoms for equilibrated structures were calculated. All-atom pair correlation functions of epoxy with $0 \%, 43 \%$ and $67 \%$ crosslinking 
density are plotted in Figure 2. Crosslinking densities were calculated from the ratio of number density of reacted sites to total number of active sites. The RDFs of neat epoxy systems at different crosslinking densities show a value of zero over short ranges $(<1-2 \AA)$ due to excluded volume effects ${ }^{62}$ and flatten out at distances beyond $8 \AA$ due to lack of long-range order. In Figure 2a, the peaks within $3.5 \AA$ are due to hydrogen and chemical bonds between atoms. Beyond $3.5 \AA$, the peaks 5 correspond to van der Waals and electrostatic interactions ${ }^{63}$. The role of inter-molecular interactions becomes significant at larger separations. As can be seen in Figure 2b, with increasing the cross linking density, the intra-molecular RDFs approach zero at longer distances (Figure $2 \mathrm{~b}$ inset). The growth of the polymer network can be traced by monitoring short and long range inter-molecular interactions. Inter-molecular RDFs of longer chains as in crosslinked network approach to unity at longer distances.

${ }_{10}$ Since new bonds are formed during crosslinking, some atom types (Figure 1d) are changed and can be specifically monitored. For instance, $\mathrm{C}-\mathrm{O}$ bonds in the epoxide ring break and result in the formation of hydroxyl $(-\mathrm{OH})$ and methyl $(-\mathrm{CH})$ groups. The number density of $o 2 e$ and $c 4 o$ atom types also decrease, while $o 2 h$ and $c 4$ atom types increase. In addition, the types of nitrogen atoms also differ: the creation of C-N bonds between EPON862 and TETA molecules changes the atom types from $n 3 h 2$ and $n 3 h 1$ to $n 3 h 1$ and $n 3$, respectively.

${ }_{15}$ Figure 3a and Figure 3b show the inter- and intra- molecular RDFs of N-O atoms, respectively. Four sharp peaks appear in $\mathrm{g}_{\text {intra }}(r)$ at around $2.3 \AA$ (N-C-O sequence), $3.1 \AA$ (N-C-O-C-O atom sequence), $3.8 \AA$ (O-C-N-C-O sequence) and $4.4 \AA$ ( $\mathrm{N}$ $\mathrm{C}-\mathrm{N}-\mathrm{C}-\mathrm{O}$ sequence) in both crosslinked systems and no peak in uncrosslinked epoxy systems since there is no linkage between $\mathrm{N}$ and $\mathrm{O}$ prior to crosslinking (Figure $3 \mathrm{a}$ ). In addition, these peaks are enhanced in intensity and slightly shifted to larger distances with increasing crosslinking density; the effect is particularly enhanced for the peaks at 2.3 and $4.4 \AA$, zoomed 20 in as insets * and **, respectively. The first of these corresponds to direct bond formation, and the area under the curves which increases by a factor of $\sim 1.7$ reflect the increase from 43 to $67 \%$ crosslinking (a $\sim 1.6$-fold increase). That the curves shift to the right as more bonds are formed attests to the fact that bonds between farther atom pairs are tolerated. More interesting is the increase in the peak at $4.4 \AA$ which corresponds to the average distance in N-C-N-C-O atom sequence on EPON-TETA crosslinked unit. The area under the curves increase by a factor of $\sim 2.2$ in this case, implying that the formation 25 of a crosslink increases the likelihood of crosslinking on that neighbour by arranging the reaction centres in close proximity. Inter-molecular RDFs between $\mathrm{N}$ and $\mathrm{O}$ atoms (Figure $3 \mathrm{~b}$ ), display the first peak at around $3.1 \AA$, corresponding to hydrogen bonds between polar atoms, and the second peak at around $4.9 \AA$, corresponding to non-bonded inter-chain interactions (van der Waals and electrostatics $)^{9}$. Since new bonds are formed in the system, intermolecular interactions reduce and $g_{\text {inter }}(r)$ peaks are suppressed with increasing crosslinking density.

30 The intra-molecular RDFs of N-C pairs are plotted in Figure $3 \mathrm{c}$ for separation distance up to $5 \AA$ A. The first major peak at around $1.5 \AA$ belongs to the $\mathrm{C}-\mathrm{N}$ bond where the peak intensity increases with conversion rate. This also indicates the formation of new N-C bonds upon crosslinking. The second peak at $2.6 \AA$ corresponds to C-C-N atom sequence $(c 43 o-c 4-$ $n 3 h 2$ (or $n 3 h 1$ or $n 3$ ) or $c 4-c 4-n 3 h 2$ (or $n 3 h 1$ or $n 3))^{9}$. Due to new linkages between C-N atoms, the number density of nonadjacent $\mathrm{C}-\mathrm{N}$ pairs increases and so does the intensity of the second peak, as seen in Figure 3c. Inter-molecular correlations ${ }_{35}$ between $\mathrm{N}$ and $\mathrm{C}$ atoms are given in Figure $3 \mathrm{~d}$, as in $\mathrm{N}-\mathrm{O}$ intermolecular interactions, $g_{\text {inter }}(r)$ peaks are depressed with increasing crosslinking density. These two intermolecular interactions also address the affinity of crosslinking. 

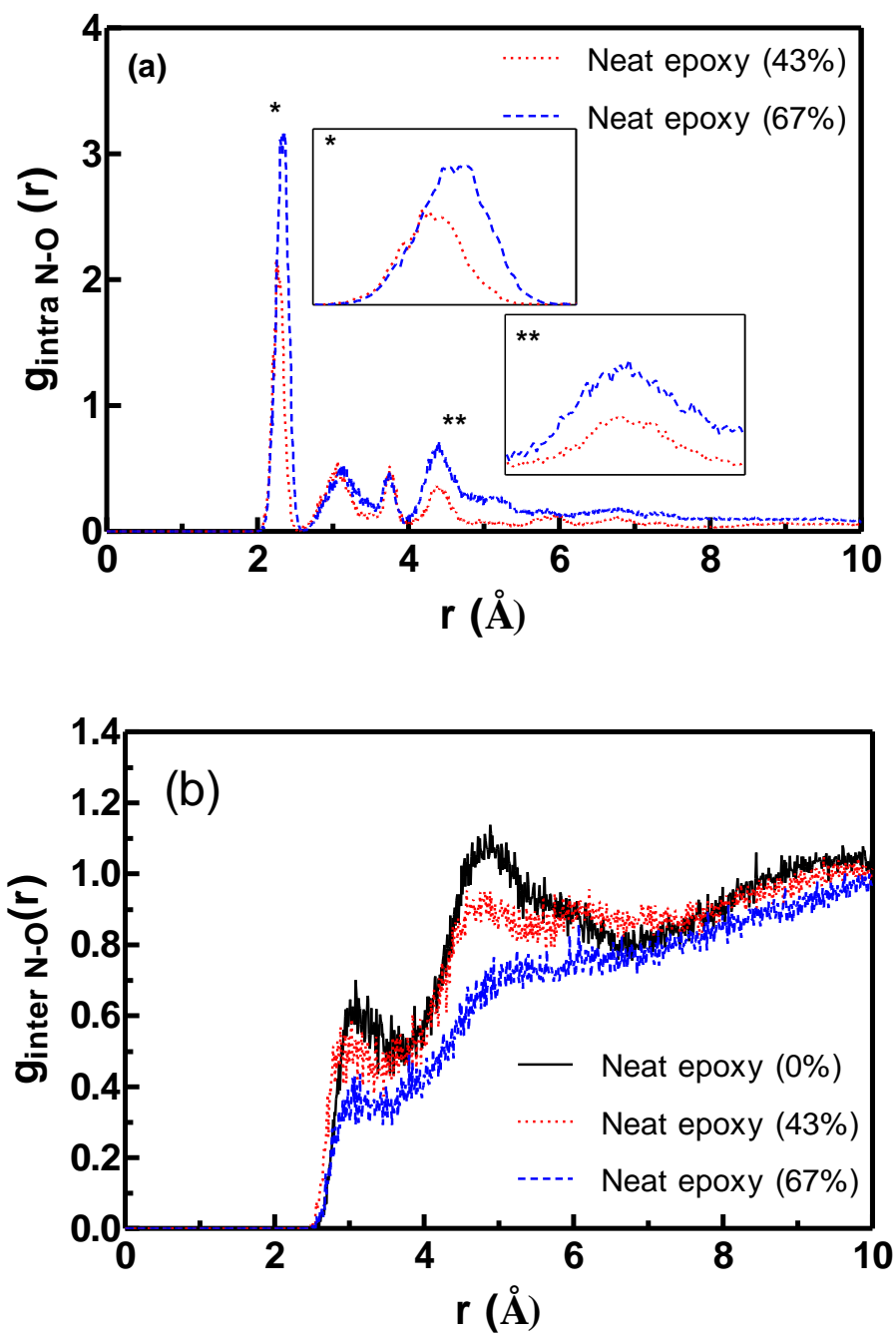

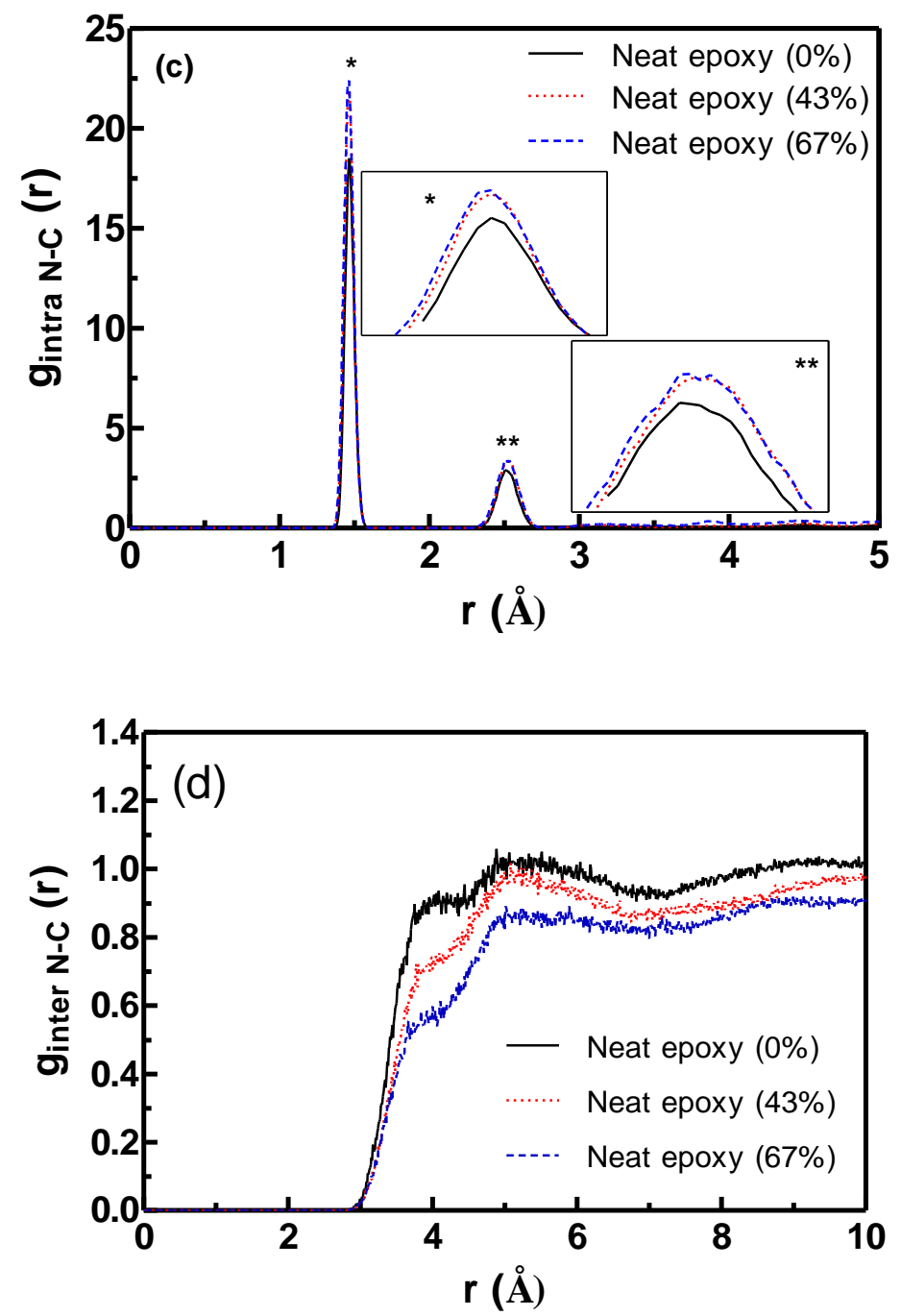

Fig. 3 (a) Intra- and (b) inter-molecular $\mathrm{N}-\mathrm{O}$ pair correlation functions, at, $43 \%$ and $67 \%$ cross linking densities (c) Intra- and (d) intermolecular $\mathrm{N}-\mathrm{C}$ pair correlation functions, at $0 \%, 43 \%$ and $67 \%$ cross linking densities

\section{s 3.2 Local Structure Evolution of Carbon Nanotube Reinforced Crosslinked Networks}

Earlier computational work in the literature ${ }^{22,29,31}$ constructed CNT reinforced epoxy networks by assuming that the structure of polymer is the same as in neat epoxy. However, the effect of CNTs or other nanofillers on crosslinking has been less studied. Our experimental studies ${ }^{64}$ and observations have shown that incorporation of CNTs considerably decreases crosslinking density in epoxy. This effect may be seen by monitoring the reduced glass transition temperature of composites ${ }_{10}\left(T_{g}\right)^{64}$ or by directly determining crosslinking density by the Soxhlet extraction technique. The lack of attractive interactions at the interface can lead to depression of $T_{g}$ relative to bulk values ${ }^{65}$. In addition, nanoscale inclusions such as CNTs may alter curing kinetics which would result in further changes in segmental mobility and a depression of $T_{g}$. Thus, they would impede attaining a consistent crosslink density for the standard curing cycle ${ }^{66}$. Our experimental studies have demonstrated that the incorporation of MWCNTs into the polymer matrix reduced crosslinking density and $T_{g}$ from $73{ }^{\circ} \mathrm{C}$ to $68{ }^{\circ} \mathrm{C}$ (See

${ }_{15}$ Figure S4 and ref $^{53}$ ). Herein, we investigate how CNTs affect the crosslinking reaction in epoxy matrix at the molecular scale. Figure 4a shows intermolecular correlation function of $\mathrm{N}-\mathrm{C}$ pairs at $0 \%$ crosslinking density for reinforced and unreinforced systems. In the presence of CNTs, intermolecular interactions are reduced, and $\mathrm{g}_{\text {inter }}(r)$ peaks are decreased. We observe the same behaviour with increasing crosslinking density, which relates to the affinity of crosslinking in the system. Figure $4 \mathrm{~b}$ provides a more detailed description using the intermolecular correlation function of $\mathrm{N}-\mathrm{C} 4 \mathrm{O}$ pairs at $0 \%$ crosslinking density. 20 The $-C 4 O$ atom is preferred since it is connected to $O$ in the epoxide ring. The $\mathrm{g}_{\text {inter }}(\mathrm{r})$ peak at around $3.8 \AA$ is found within the cut-off distance for crosslinking reaction. The intensity of the first $g_{\text {inter }}$ peak for reinforced system is lower than that for neat epoxy. To provide insight as to why the degree of crosslinking is reduced in the presence of CNTs (e.g. crosslinking 
degree from $43 \%$ to $27 \%$ for two reactive sites and from $67 \%$ to $56 \%$ for four reactive sites), we have studied intermolecular correlation function of $N-c 3 a$ pairs at different crosslinking densities. The aromatic groups as in epoxy molecule can physisorb onto the walls of carbon nanotubes due to $\pi-\pi$ stacking ${ }^{67}$. Therefore, we explored the interaction of $c 3 a$ atoms in epoxy and carbon nanotube with hardener (atom type $N$ ). Relatively strong interaction of the epoxy $(c 3 a)$-CNT $(c 3 a)$ pair ${ }_{5}$ may impede crosslinking reaction since epoxy $(c 3 a)$-TETA $(N)$ is not solely dominant in reaction cut-off distance. While increasing crosslinking density, intermolecular correlation function of epoxy $(c 3 a)$ - CNT $(c 3 a)$ pairs slightly decreases due to the effect of this interaction, as seen in Figure 5. Crosslinked molecules of unreinforced and reinforced epoxy systems at different conversion rates and the number density of different type of crosslinked chains are shown in Figure 6. Figure 6a and Figure $6 \mathrm{~b}$ suggest that the crosslinking degree would be enhanced by increasing the number of reactive sites through 10 assigning both $n 3 h 2$ and $n 3 h 1$ atoms as reactive pairs. Besides, it is clear that increasing the number of reactive atoms leads to much complex crosslinked units and unreacted TETA molecules are totally eliminated when crosslinking reaction is completed. In CNTs reinforced systems, the degree of crosslinking is reduced while observing simpler crosslinked geometries are observed compared to neat crosslinked epoxy systems (Figure 6c and Figure 6d). More surprisingly, unreacted TETA molecules are detected in the presence of CNTs, explaining the reduced crosslinking degree (Figure 6c and 6d).

${ }_{15}$ Figure 7a shows the inter-molecular RDFs of N-O atoms in reinforced systems. While the location and intensity of the first peak does not differ, the second peak which corresponds to non-bonded interchain interactions is reduced compared to that for neat epoxy system with $0 \%$ degree of crosslinking. Thus, incorporation of CNTs lessen the intermolecular interactions between TETA and epoxy. In addition, inter-molecular correlations between $\mathrm{N}$ and $\mathrm{C}$ atoms are given in Figure 7b; as in neat epoxy systems, $g_{\text {inter }}(\mathrm{r})$ peaks are depressed with increasing crosslinking density, explaining the affinity of crosslinking in the 20 presence of CNTs. The intra-molecular RDFs of N-C pairs in reinforced epoxy systems are plotted in Figure 7c for separation distance up to $5 \AA$. The new formed N-C bonds upon crosslinking can be monitored from the first major peak around $1.5 \AA$. 

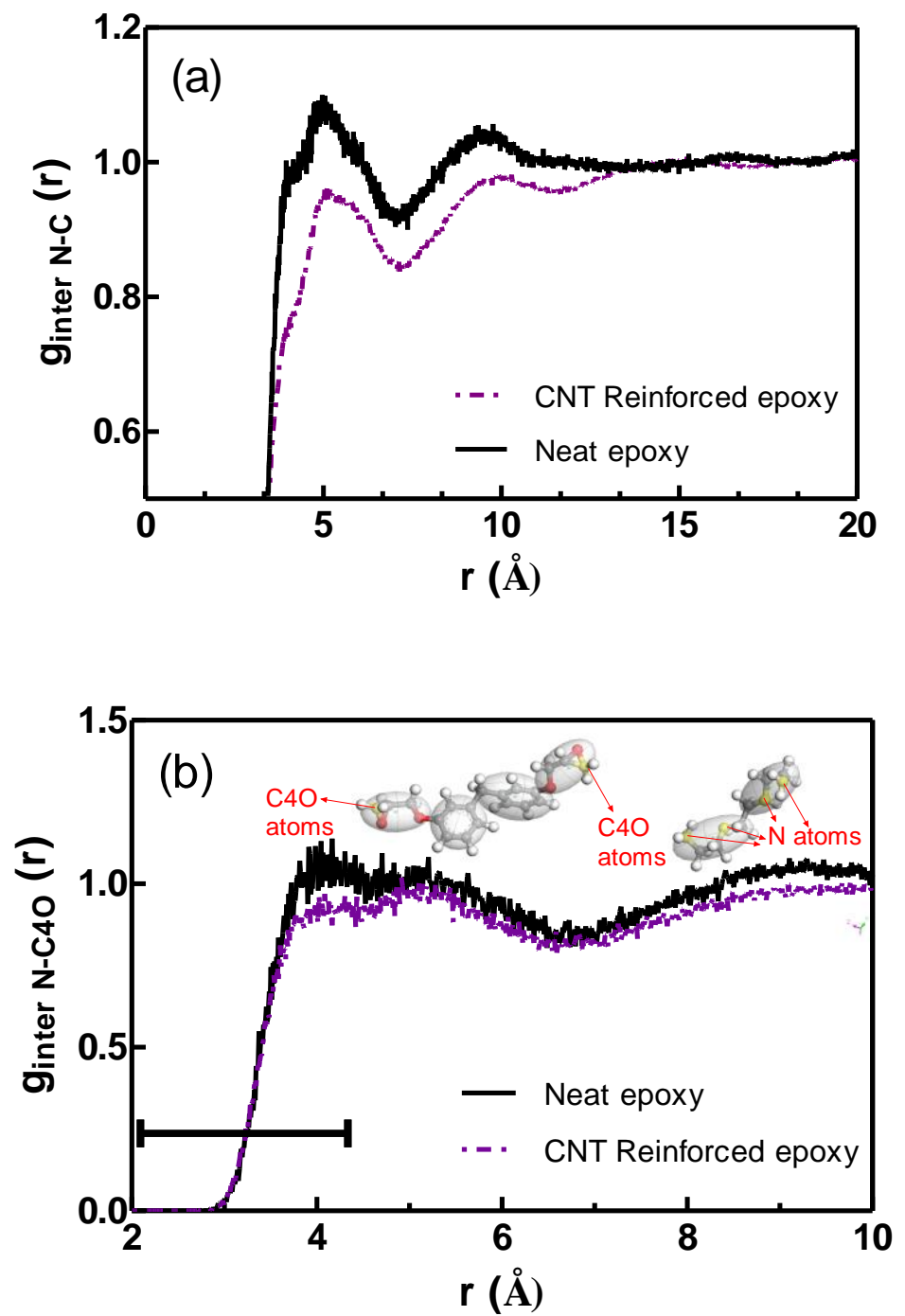

Fig. 4 Inter- molecular correlation functions of: (a) $\mathrm{N}-\mathrm{C}$ pairs and (b) $\mathrm{N}-\mathrm{C} 4 \mathrm{O}$ pairs in neat epoxy and CNT reinforced epoxy systems at $0 \%$ crosslinking densities 

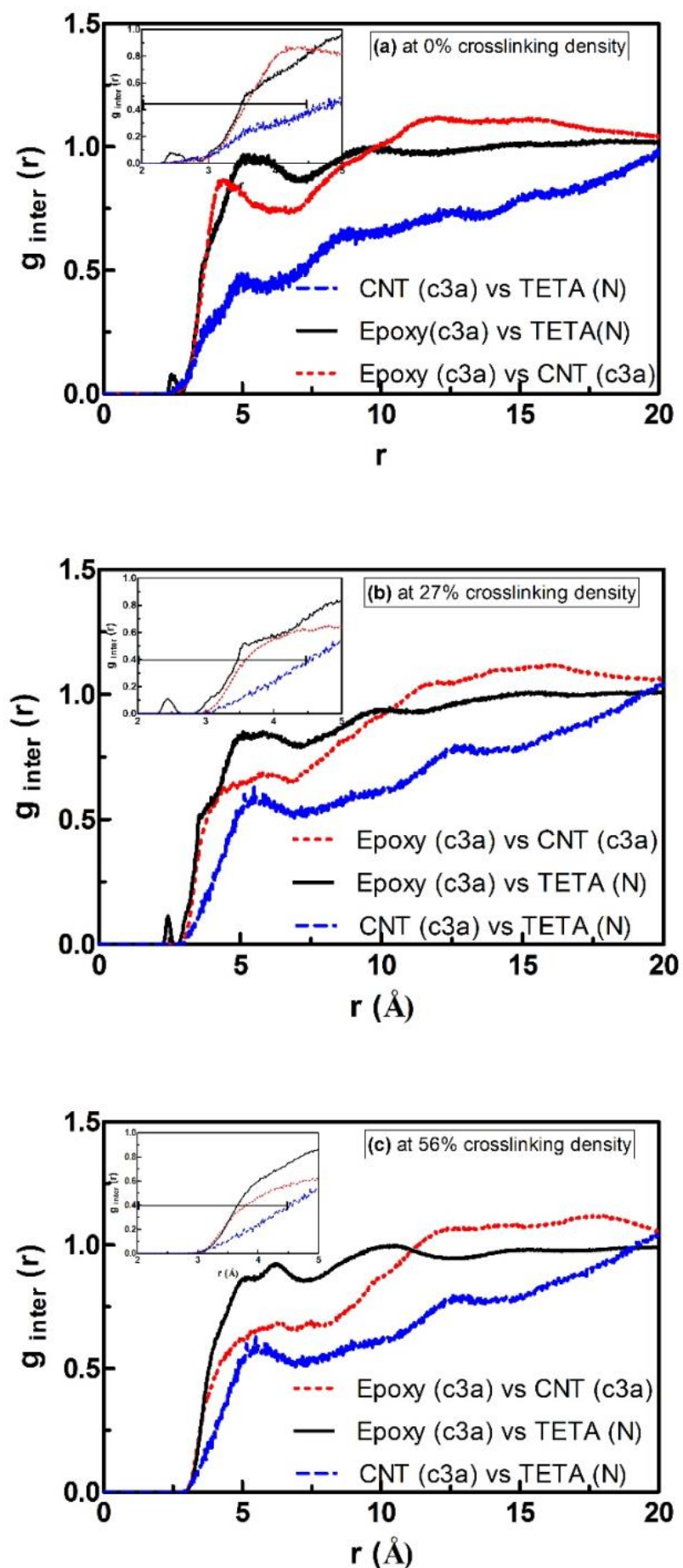

Fig 5 Inter-molecular epoxy (c3a)-CNT (c3a), epoxy (c3a)-TETA (N), CNT (c3a)- TETA (N) pair correlation functions of CNT reinforced epoxy systems (a) at $0 \%$ crosslinking density (b) at $27 \%$ crosslinking density and (c) at $56 \%$ crosslinking density 
(a) Neat epoxy (43\%)
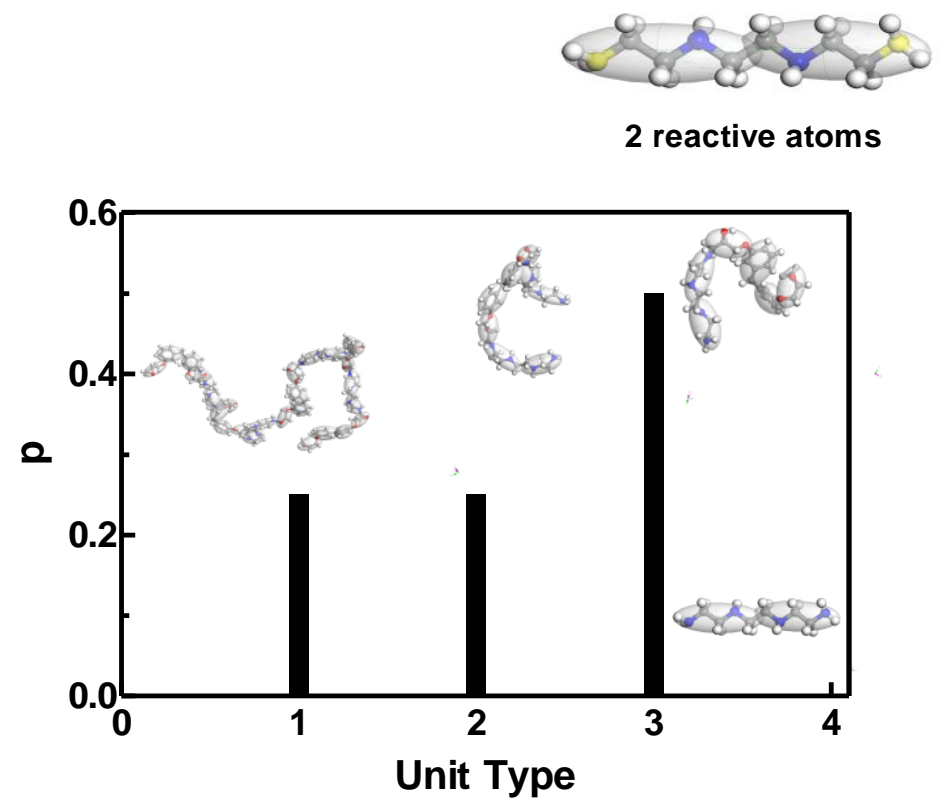

(b) Neat epoxy (67\%)

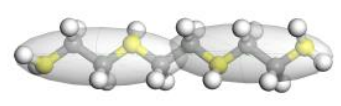

4 reactive atoms

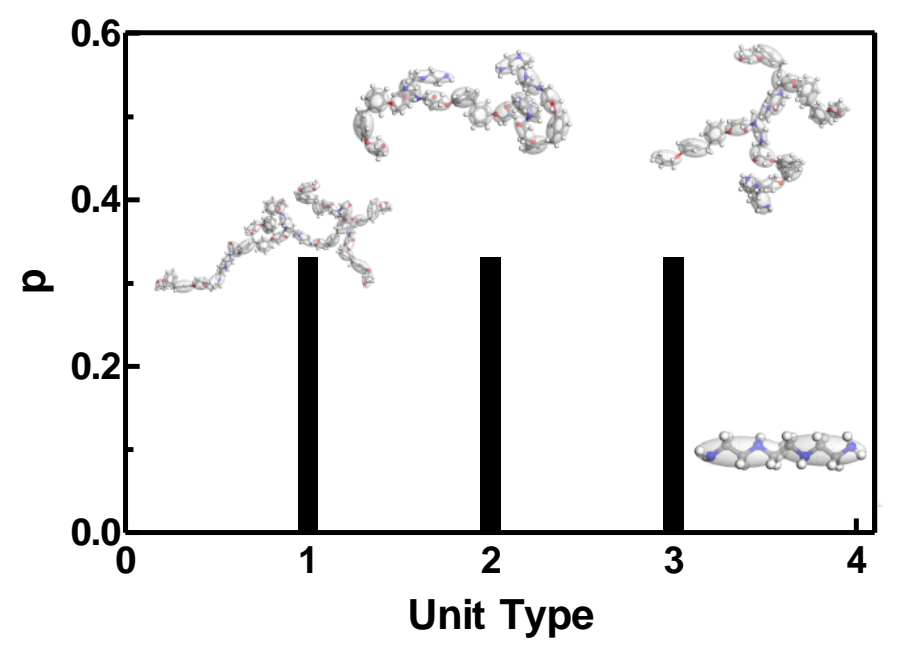


(c) CNT Reinforced epoxy (27\%)

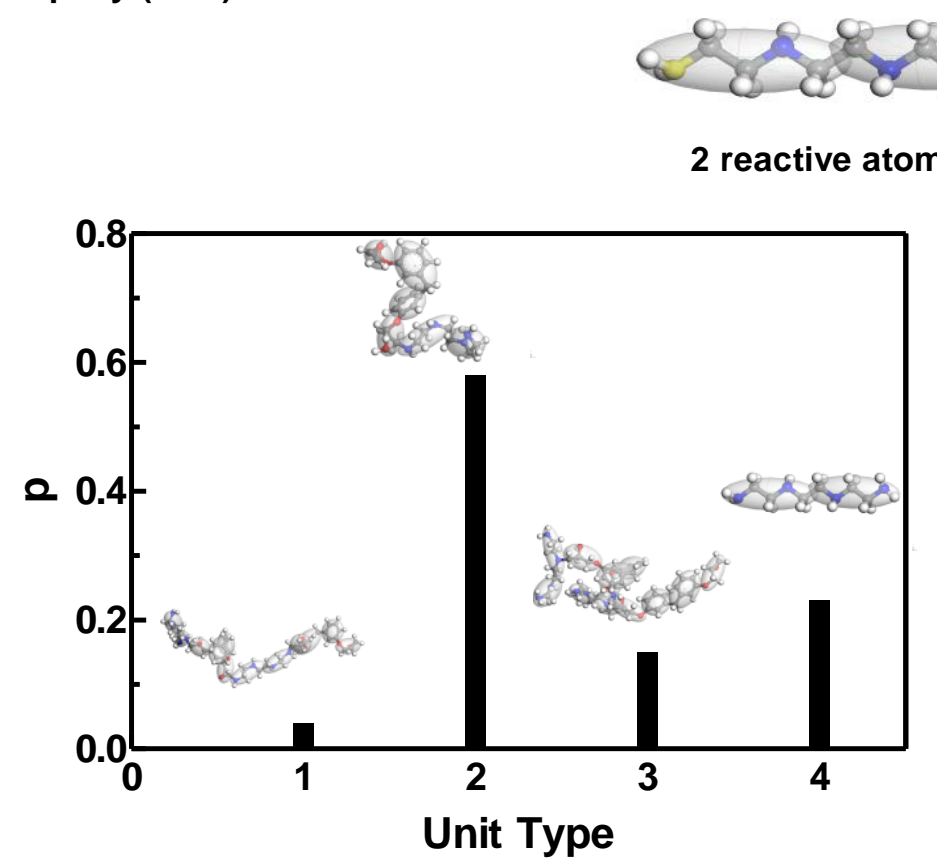

(d) CNT Reinforced epoxy (56\%)

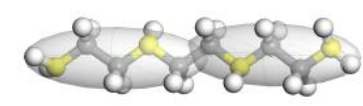

4 reactive atoms

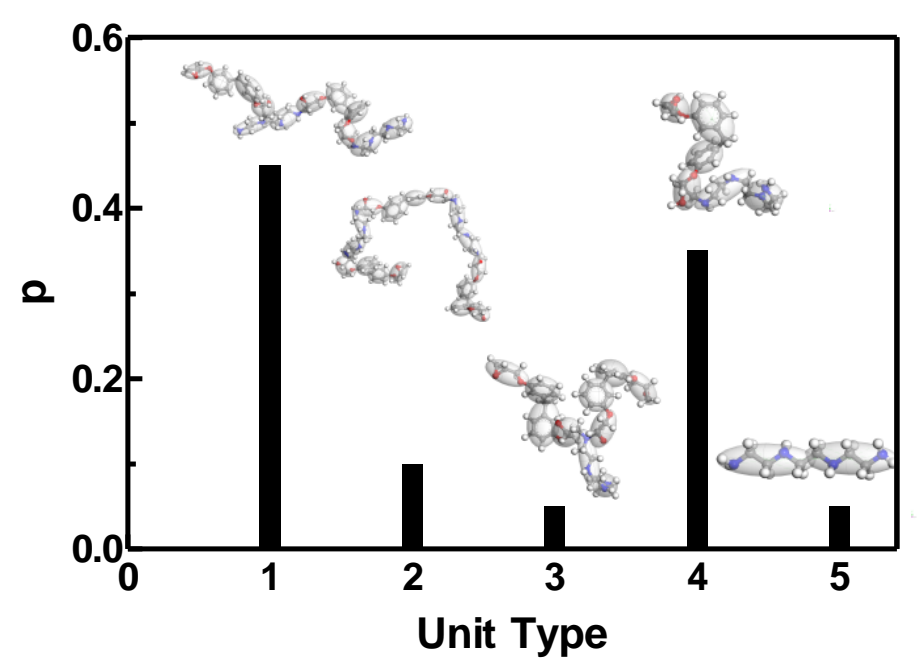

Fig. 6 Representative EPON862-TETA crosslinked chains created in neat epoxy systems (a) at 43\% and (b) 67\% crosslinking densities and CNT reinforced epoxies (c) at $27 \%$ and (b) $56 \%$ crosslinking densities 

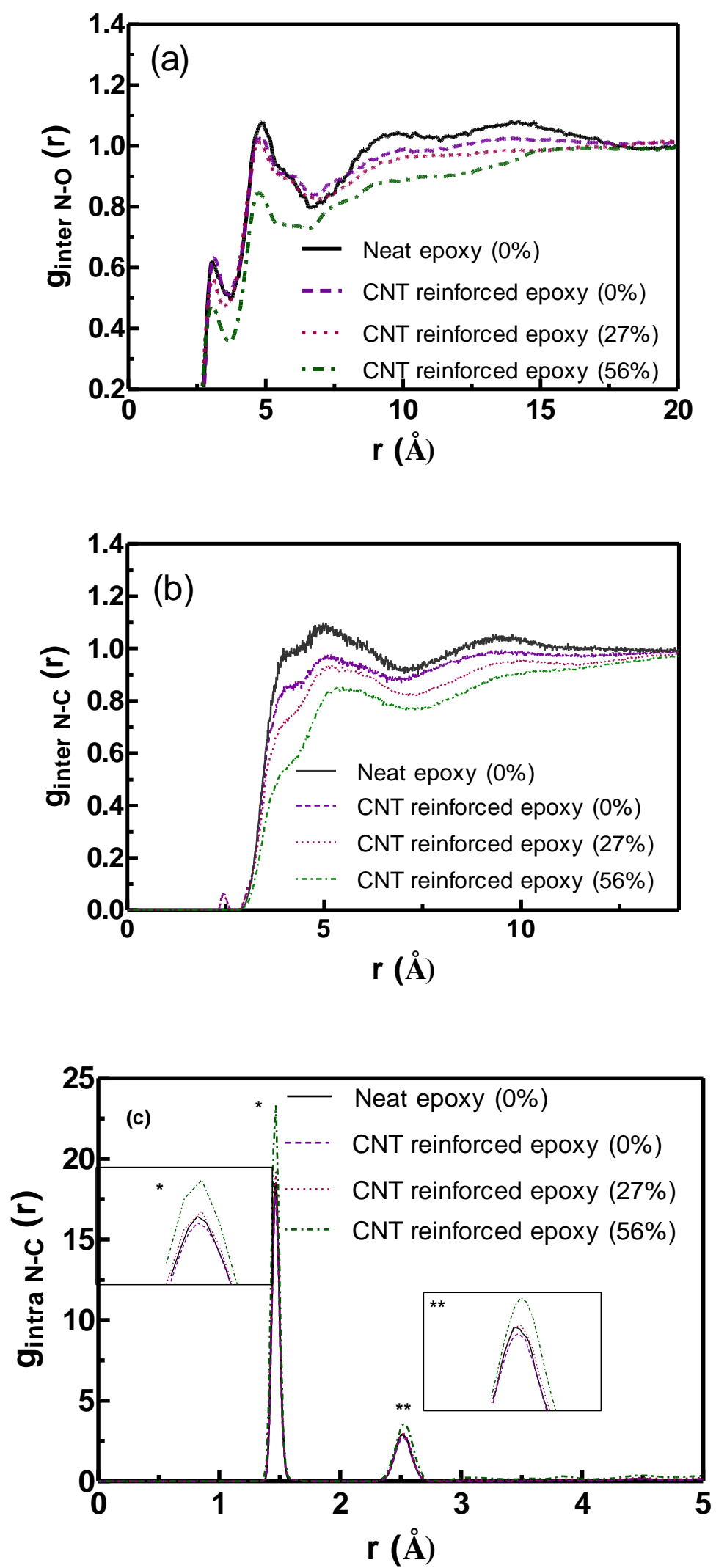

Fig. 7 (a) Inter-molecular- $\mathrm{N}-\mathrm{O}$ pair correlation functions, (b) inter-molecular and (c) intra-molecular $\mathrm{N}-\mathrm{C}$ pair correlation functions of neat epoxy (0\%) and CNT reinforced epoxy systems at $0 \%, 27 \%$ and $56 \%$ crosslinking densities. 


\subsection{Mechanical Properties of Neat Epoxy and CNTs Reinforced Systems at Different Crosslinking Densities}

To calculate the mechanical behaviour of the neat epoxy system and monitor the effects of crosslinking, we utilized reversemapped trajectories of both neat and reinforced epoxy systems. The mechanical tests described below were carried out to determine the stress-strain behavior in the normal directions $(x, y)$. Here, we first investigate the change in compressive/tensile ${ }_{5}$ strength and modulus during the process of curing reaction in neat epoxy systems. In Figure 8a-d, it is clear that both compressive/tensile strength and elastic modulus have an apparent dependence on the conversion of reactant. As the curing reaction proceeds, the compressive/tensile strength ( $0.2 \%$ strain offset) and compressive/elastic modulus (slopes of the stressstrain curves) both increase; these are listed in Table 2. Figure $8 \mathrm{~b}$ also shows true stress-strain curves of epoxy matrices in compression direction. The highest compressive strength and elastic moduli are observed in $67 \%$ crosslinked epoxy systems 10 (See Figure 8a, Figure 8b and Table 2). As also seen in Table 2, crosslinking plays a significant role in enhancing mechanical properties, as might be expected. As the reaction proceeds, more linkages are formed among epoxy and hardener molecules, reducing chain mobility. Thus, compressive strength and modulus gradually increase with an increasing extent of reaction. Figure $8 \mathrm{c}$ reveals stress-strain behaviour in another normal direction, $x$, where is subjected to tensile loading. Figure 8d describes true stress-strain curves in tensile loading. New linkages in the matrix may lead to anisotropy. Therefore, as in ${ }_{15}$ Figure $8 \mathrm{~b}$ and Figure 8d, normal stresses in tensile and compressive loading direction up to $0.2 \%$ strain is also calculated and yield strength $(0.2 \%$ offset) and compressive/elastic modulus in both normal directions are reported. There is slight difference between the elastic moduli in the tensile ( $x$ and $z$ ) directions where at higher conversion rates this becomes much clearer due to the contribution of new linkages. Besides, it is found that compressive strength in yy direction for 3D epoxy networks is higher than their tensile strength. Overall, material constants of unreinforced epoxy systems are strongly dependent on ${ }_{20}$ conversion rate and in line with computational and experimental observations ${ }^{1,68-71}$. The mechanical properties evaluation via the application of incremental strains enabled the calculation of compressive and tensile yield strength ( $0.2 \%$ offset) for neat epoxy at different crosslinking densities, which has not been reported in the literature.

We next present the results due to the inclusion of CNTs into the system. CNTs are known as stiff and strong materials that would also increase mechanical properties of epoxy. On the other hand, their incorporation into thermoset matrices reduces ${ }_{25}$ crosslinking density, which has the reverse effect than stiffening. Besides, due to tubular structure and mismatches in elastic modulus in different directions, CNTs would contribute to anisotropy. First, as displayed in Figure 9a and Figure 9c, the stress-strain behavior in compressive and tensile loadings indicated enhanced mechanical response with the incorporation of CNTs. Figure 9b and Figure 9d exhibits true stress-strain curves where the contribution of CNTs on mechanical properties becomes clearer. Even though CNTs reduce the degree of crosslinking in epoxy matrix, which may adversely affect ${ }_{30}$ mechanical properties, their high strength and stiffness compensate for this effect. Figure 8a and Figure 9 display yield strength ( $0.2 \%$ strain) and elastic modulus in compression and tensile direction, respectively. It is clear that embedding CNTs into epoxy systems increase both modulus and strength of the polymer network. ${ }^{68}$

Table 2 Mechanical properties of neat and CNTs reinforced systems at different conversion rates, the calculations are displayed in Figure 35 S5-S8.

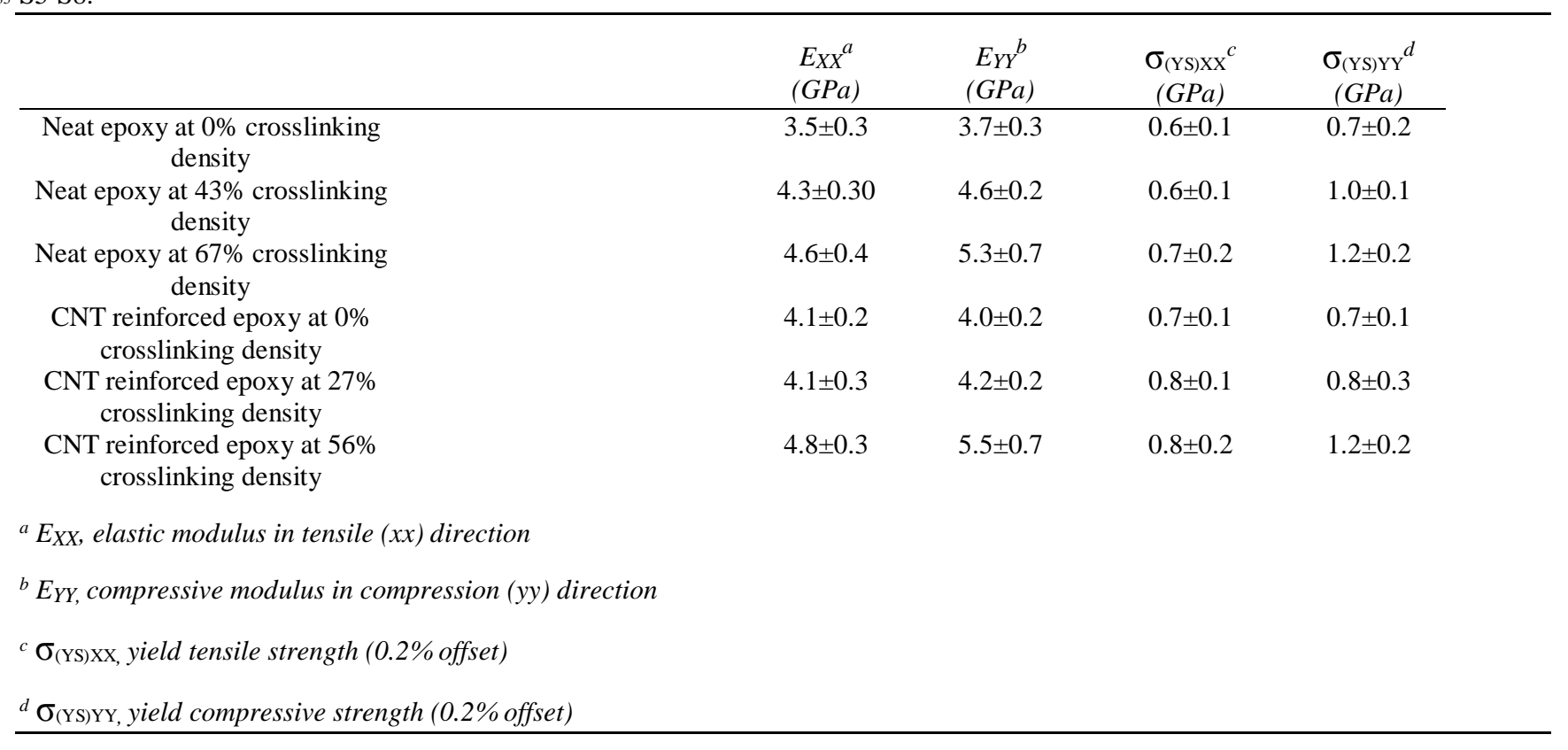



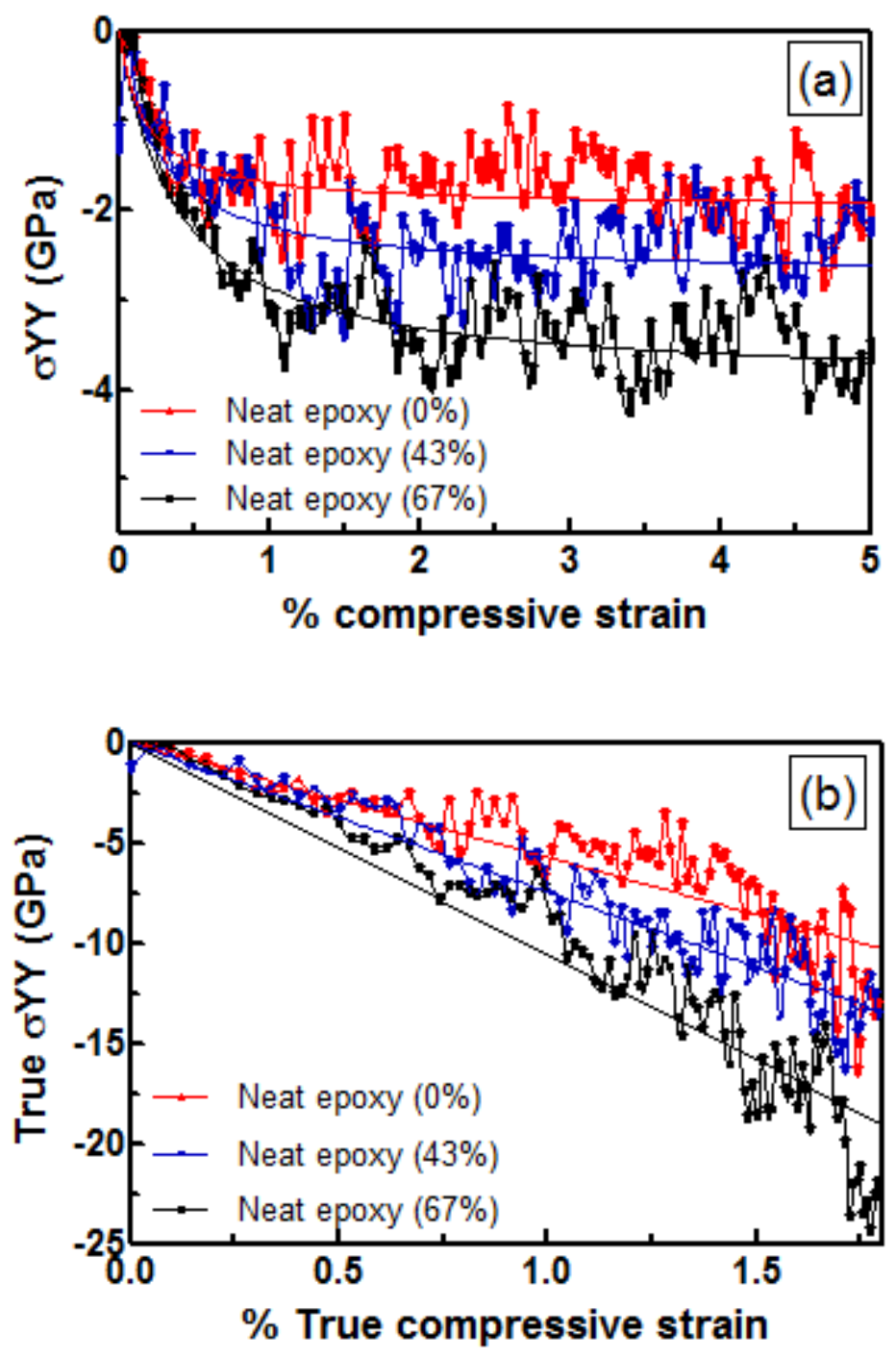

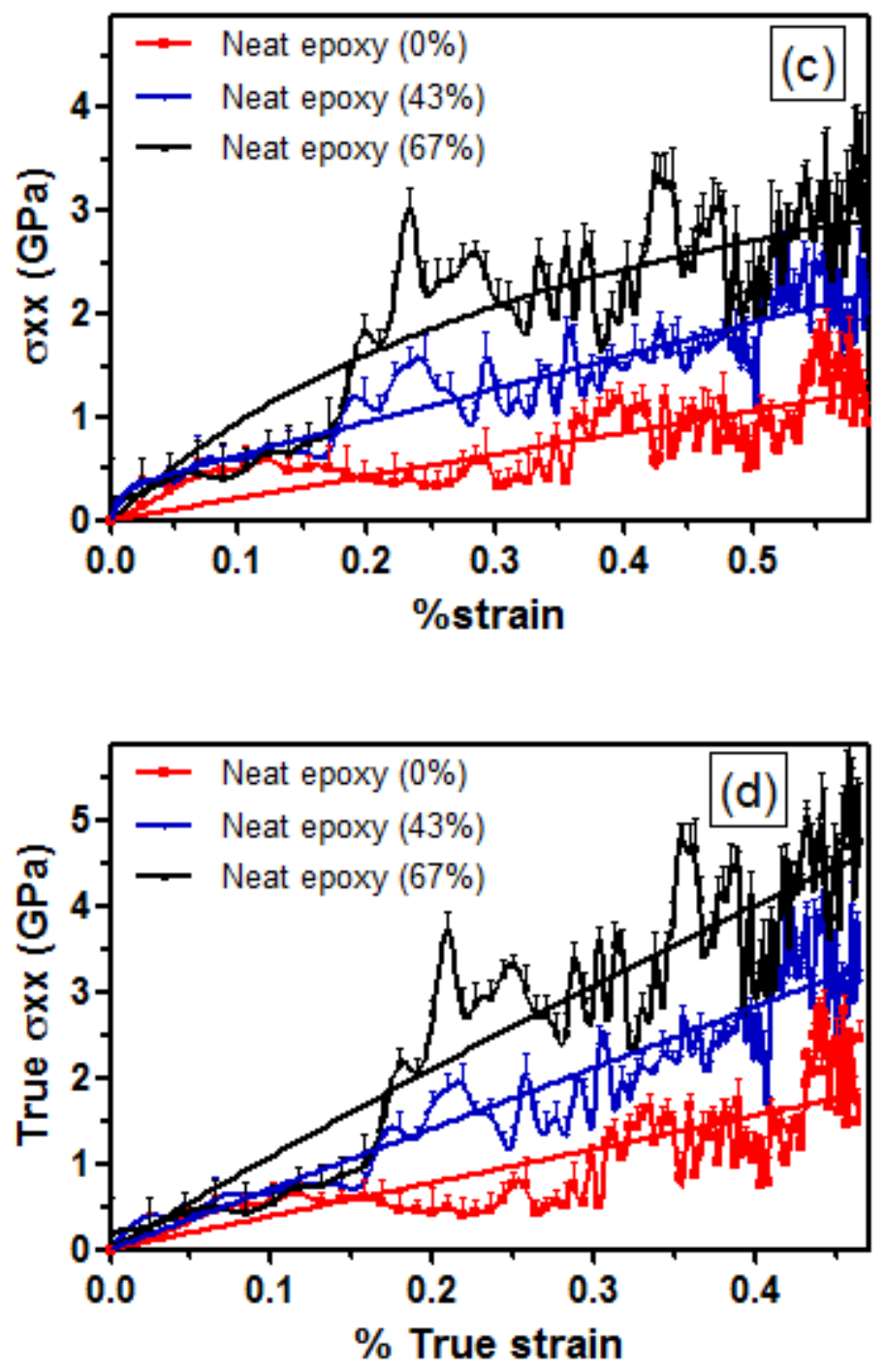

Fig. $8 \mathrm{MD}$ simulated (a) $\sigma_{Y Y}$ stress-strain curves, (b) True $\sigma_{Y Y}$ stress-strain curves, (c) $\sigma_{X X}$ stress-strain curves and (d) True $\sigma_{X X}$ stressstrain curves of curves of neat epoxy resins at $0 \%, 43 \%$ and $67 \%$ crosslinking densities 

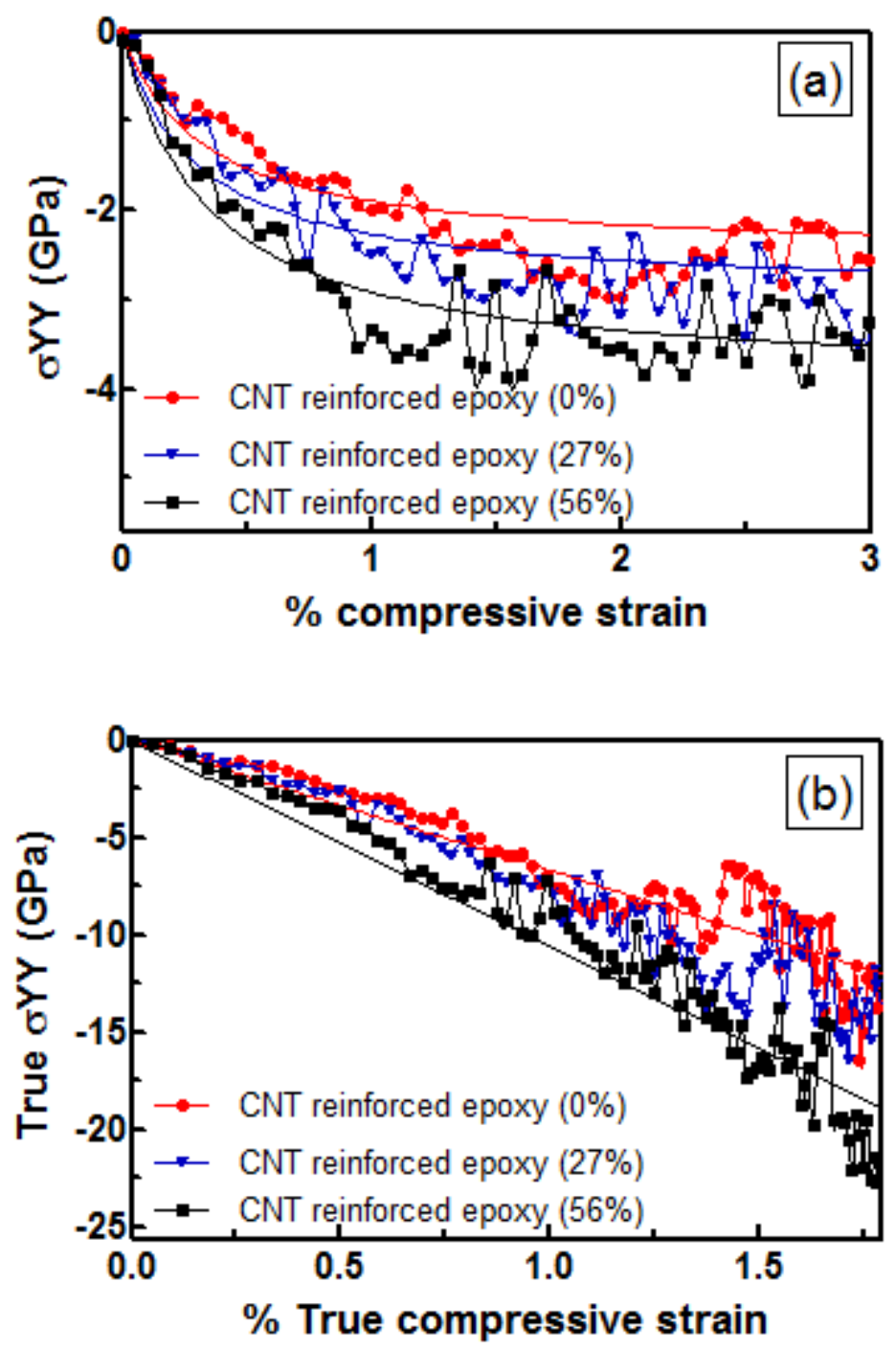

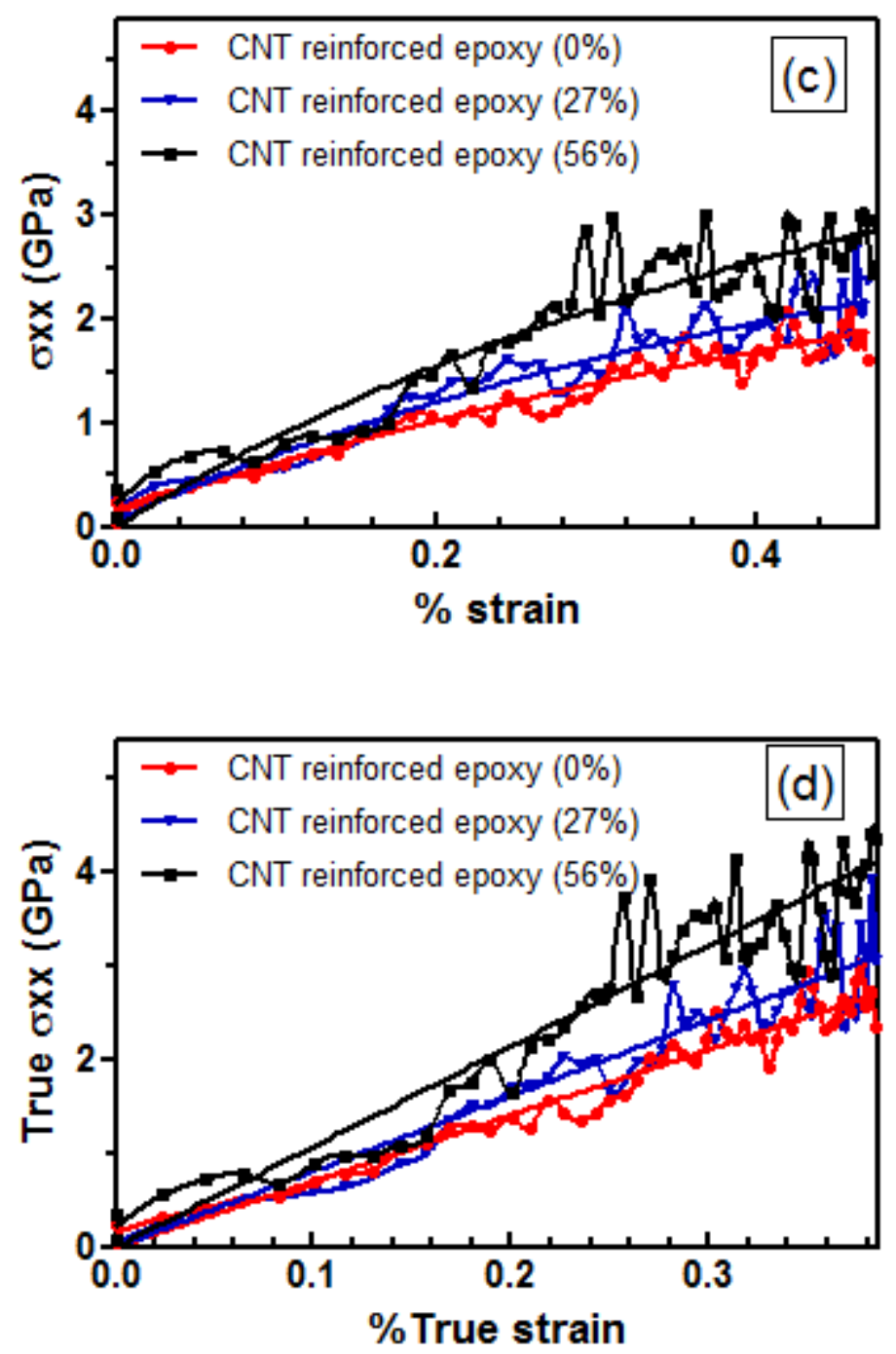

Fig. 9 MD simulated (a) $\sigma_{Y Y}$ stress-strain curves, (b) True $\sigma_{Y Y}$ stress-strain curves, (c) $\sigma_{X X}$ stress-strain curves and (d) True $\sigma_{X X}$ stressstrain curves of CNT reinforced epoxy systems at $0 \%, 27 \%$ and $56 \%$ crosslinking densities.

\section{Conclusions}

We have developed a computational strategy for simulating the formation of crosslinked neat and CNTs reinforced polymer networks and predicting their physical and mechanical properties. This methodology based on multiscale simulations involves the following four separate procedures: (i) coarse-gaining of epoxy monomers and CNTs, (ii) reverse mapping of the coarse-grained model to a fully atomistic 10 representation and its further equilibration and refinement (iii) crosslinking the monomers (iv) simulation of the atomistic model through classical molecular dynamics (MD) and mechanical testing simulations. To the best of our knowledge, periodic structures such as carbon nanotubes are coarse-grained as rigid rods and then reverse-mapped for the first time. Local structural evolution is monitored to reveal the effect of CNTs on crosslinking reaction. Our results indicate clearly that, both the elastic moduli (slopes of the stress-strain curves) and compressive strength (at $0.2 \%$ offset) of the reinforced composites increases remarkably. Moreover, both compressive/tensile strength and 15 modulus gradually increase with an increase of conversion, both in neat and reinforced epoxy systems. These findings are in line with experimental observations ${ }^{68}$; we note that not only qualitative but also quantitative agreement is obtained whereby the calculated moduli are on the order of a few GPa at room temperature. Due to the atomistic scale representation in this work, we are now able to trace the origin of the measurements to interactions at the interface of the nanocomposite. In particular, the breaking of epoxide ring and the formation of N-C-O linkages play an important role in the enhanced mechanical properties (Figure 3 and Figure 4). 


\section{Acknowledgement}

The work presented here was supported by TUBITAK 3001 (Project No: 213M618). The author E.O-Y also thanks Melih ${ }_{5}$ Papila for conducting DMA analysis and the discussion on crosslinking densities and $\mathrm{T}_{\mathrm{g}}$.

\section{Supporting information is available.}

*Corresponding Author: Department of Textile Engineering, Faculty of Textile Technologies and Design, Istanbul Technical University, ${ }_{10}$ Beyoglu, Turkey; Phone: 9021229313 00/2497 Fax: +90 21225188 29 E-mail: ozdenyenigun@itu.edu.tr

\section{References}

1. H. B. Fan and M. M. F. Yuen, Polymer, 2007, 48, 2174-2178.

2. P. V. Komarov, Y. T. Chiu, S. M. Chen, P. G. Khalatur and P. Reineker, Macromolecules, 2007, 40, 8104-8113.

15 3. C. Y. Li, E. Jaramillo and A. Strachan, Polymer, 2013, 54, 881-890.

4. C. Y. Li, G. A. Medvedev, E. W. Lee, J. Kim, J. M. Caruthers and A. Strachan, Polymer, 2012, 53, 4222-4230.

5. Y. C. Lin and X. Chen, Chem Phys Lett, 2005, 412, 322-326.

6. M. Onodera, T. Okabe, M. Hasimoto and K. Yoshioka, Proceedings of the American Society for Composites, 2013.

7. F. Sawa and T. Imai, Proceedings of 2014 International Symposium on Electrical Insulating Materials (Iseim 2014), 2014, $327-329$.

20 8. E. Serrano, G. Kortaberria, P. Arruti, A. Tercjak and I. Mondragon, Eur Polym J, 2009, 45, 1046-1057.

9. A. Shokuhfar and B. Arab, J Mol Model, 2013, 19, 3719-3731.

10. J. L. Tack and D. M. Ford, J Mol Graph Model, 2008, 26, 1269-1275.

11. L. H. Tam and D. Lau, Rsc Adv, 2014, 4, 33074-33081.

12. V. Varshney, S. S. Patnaik, A. K. Roy and B. L. Farmer, Macromolecules, 2008, 41, 6837-6842.

25 13. V. Varshney, S. S. Patnaik, A. K. Roy and B. L. Farmer, Polymer, 2009, 50, 3378-3385.

14. C. F. Wu and W. J. Xu, Polymer, 2007, 48, 5802-5812.

15. B. Wunderle, E. Dermitzaki, O. Holck, J. Bauer, H. Walter, Q. Shaik, K. Ratzke, F. Faupel, B. Michel and H. Reichl, Microelectron Reliab, 2010, 50, 900-909.

16. D. R. Xin and Q. Han, J Mol Model, 2015, 21.

30 17. L. Yao, Chinese J Polym Sci, 2015, 33, 465-474.

18. L. Yao, Y. Xia and Q. Zhou, J Adhes Sci Technol, 2015, 29, 753-766.

19. N. Iwamoto, Microelectron Reliab, 2011, 51, 1035-1045.

20. H. Yagyu, Y. Hirai, A. Uesugi, Y. Makino, K. Sugano, T. Tsuchiya and O. Tabata, Polymer, 2012, 53, 4834-4842.

21. J. Choi, S. Yu, S. Yang and M. Cho, Polymer, 2011, 52, 5197-5203.

35 22. J. C. Huang and C. Y. Chiu, Polym Polym Compos, 2011, 19, 377-382.

23. D. H. Kim and H. S. Kim, Composites Science and Technology, 2014, 101, 110-120.

24. R. Kotsilkova, D. Fragiadakis and P. Pissis, J Polym Sci Pol Phys, 2005, 43, 522-533.

25. B. Mortazavi, S. Ahzi, J. Bardon, A. Laachachi and D. Ruch, Tms2011 Supplemental Proceedings, Vol 2: Materials Fabrication, Properties, Characterization, and Modeling, 2011, 117-120.

40 26. B. Mortazavi, O. Benzerara, H. Meyer, J. Bardon and S. Ahzi, Carbon, 2013, 60, 356-365.

27. M. M. Shokrieh, M. Esmkhani, Z. Shokrieh and Z. Zhao, Comp Mater Sci, 2014, 92, 444-450.

28. M. M. Shokrieh, Z. Shokrieh and S. M. Hashemianzadeh, Mater Design, 2014, 64, 96-101.

29. B. S. Sindu and S. Sasmal, Comp Mater Sci, 2015, 96, 146-158.

30. G. Sodeifian, H. R. Nikooamal and A. A. Yousefi, J Polym Res, 2012, 19.

45 31. Q. L. Xiong, S. A. Meguid, Y. Wang and G. J. Weng, Mech Mater, 2015, 85, 38-46.

32. S. R. Yang, F. Gao and J. M. Qu, Elec Comp C, 2011, 1110-1114.

33. H. W. Cui, D. S. Li and Q. Fan, Int J Adhes Adhes, 2012, 35, 50-54.

34. S. R. Yang, F. Gao and J. M. Qu, Polymer, 2013, 54, 5064-5074.

35. S. R. Yang and J. M. Qu, Model Simul Mater Sc, 2014, 22.

50 36. E. Özden Yenigün, A. R. Atılgan, K. Bilge, Y. Z. Menceloğlu, C. Atılgan and M. Papila, MRS Proceedings, $2011,1304$.

37. G. Kacar, E. A. J. F. Peters and G. de With, Comp Mater Sci, 2015, 102, 68-77.

38. R. D. Groot and P. B. Warren, Journal of Chemical Physics, 1997, 107, 4423-4435.

39. J. H. Hildebrand, The Solubility of Non-Electrolytes, Reinhold, New York, 1936.

40. MATERIAL-STUDIO, San Diego2002.

55 41. ACD/ChemSketch, Toronto, ON, Canada2003. 
42. K. P. Travis, M. Bankhead, K. Good and S. L. Owens, Journal of Chemical Physics, 2007, 127.

43. J. H. Wu and D. D. L. Chung, Carbon, 2004, 42, 3039-3042.

44. P. Doruker and W. L. Mattice, Macromolecules, 1997, 30, 5520-5526.

45. A. Ghanbari, M. C. Bohm and F. Muller-Plathe, Macromolecules, 2011, 44, 5520-5526.

5 46. V. A. Harmandaris, N. P. Adhikari, N. F. A. van der Vegt and K. Kremer, Macromolecules, 2006, 39, 6708-6719.

47. G. Kacar, C. Atilgan and A. S. Ozen, Journal of Physical Chemistry C, 2010, 114, 370-382.

48. C. Peter, L. Delle Site and K. Kremer, Soft Matter, 2008, 4, 859-869.

49. C. Peter and K. Kremer, Abstracts of Papers of the American Chemical Society, 2010, 240.

50. A. J. Rzepiela, L. V. Schafer, N. Goga, H. J. Risselada, A. H. De Vries and S. J. Marrink, J Comput Chem, 2010, 31, $1333-1343$.

10 51. G. Santangelo, A. Di Matteo, F. Muller-Plathe and G. Milano, Journal of Physical Chemistry B, 2007, 111, 2765-2773.

52. T. Spyriouni, C. Tzoumanekas, D. Theodorou, F. Muller-Plathe and G. Milano, Macromolecules, 2007, 40, 3876-3885.

53. E. Ozden-Yenigun, C. Atılgan, Y. Z. Menceloğlu and M. Papila, in European Society for Composite Materials Proceedings, http://www.escm.eu.org/eccm15/data/assets/794.pdf.

54. C. Y. Li and A. Strachan, Polymer, 2010, 51, 6058-6070.

15 55. H. Sun, Journal of Physical Chemistry B, 1998, 102, 7338-7364.

56. H. C. Andersen, Journal of Chemical Physics, 1980, 72, 2384-2393.

57. H. J. C. Berendsen, J. P. M. Postma, W. F. Vangunsteren, A. Dinola and J. R. Haak, Journal of Chemical Physics, 1984, 81, 3684-3690.

58. D. N. Theodorou and U. W. Suter, Macromolecules, 1986, 19, 139-154.

59. D. Brown and J. H. R. Clarke, Macromolecules, 1991, 24, 2075-2082.

20 60. A. A. Gusev, M. M. Zehnder and U. W. Suter, Phys Rev B, 1996, 54, 1-4.

61. M. Parrinello and A. Rahman, Journal of Chemical Physics, 1982, 76, 2662-2666.

62. J. S. Bermejo and C. M. Ugarte, Macromol Theor Simul, 2009, 18, 317-327.

63. J. P. Zeng, J. Y. Zhang and X. D. Gong, Comput Theor Chem, 2011, 963, 110-114.

64. E. Ozden-Yenigun, Y. Z. Menceloglu and M. Papila, ACS Applied Materials \& Interfaces, 2012, 4, 777-784.

25 65. P. Rittigstein and J. M. Torkelson, J Polym Sci Pol Phys, 2006, 44, 2935-2943.

66. K. W. Putz, M. J. Palmeri, R. B. Cohn, R. Andrews and L. C. Brinson, Macromolecules, 2008, 41, 6752-6756.

67. F. Tournus, S. Latil, M. I. Heggie and J. C. Charlier, Phys Rev B, 2005, 72.

68. B. Kim, J. Choi, S. Yang, S. Yu and M. Cho, Polymer, 2015, 60, 186-197.

69. S. Yang, S. Yu and M. Cho, Journal of Applied Physics, 2010, 108, 056102.

30 70. S. Yu, S. Yang and M. Cho, Polymer, 2009, 50, 945-952.

71. S. C. Zunjarrao and R. P. Singh, Composites Science and Technology, 2006, 66, 2296-2305. 